\title{
Einstein spaces in warped geometries in five dimensions
}

\author{
M. Arık A. Baykalf M. C. Calık, D. Ciftci团 and Ö. Deliç\$ \\ Department of Physics, Bog̃aziçi University, 34342 Bebek, Istanbul, Turkey
}

\begin{abstract}
We investigate five dimensional Einstein spaces in warped geometries from the point of view of the four dimensional physically relevant Robertson-Walker-Friedman cosmological metric and the Schwarzschild metric. We show that a four-dimensional cosmology with a closed spacelike section and a cosmological constant can be imbedded into five-dimensional flat space-time.
\end{abstract}

PACS numbers: 98.80.Cq, 98.70.Vc.

The general theory of relativity is an experimentally well-tested theory. Among these tests, the Schwarzschild solution has played a central role. For the cosmological solutions, however, the situation is beginning to clarify with the accumulation of relevant astrophysical data. On the one hand, a simple, consistent, logical cosmology requires a spatially maximally symmetric RobertsonWalker-Friedman cosmology with closed spacelike sections $(k=1)$. Recent observational evidence shows that we live in an expanding closed universe with positive cosmological constant [1]. The maximally symmetric Einstein de-Sitter solutions are good prototypes of such space-times since they include the cosmological constant. However, the existence of the cosmological constant is one of the deep mysteries in cosmology.

Since the Kaluza-Klein idea 2], there have been many theories suggesting that the universe may have more than four dimensions. Nowadays, the idea that our universe may be a three-brane embedded in five dimensional universe is very popular $[3]-[5]$. For a recent review see $[6]$.

The recent interest in the Randall-Sundrum [4]- $[5]$ and related scenarios has brought into consideration warped geometries such that four dimensional spacetime metric is multiplied by a warp factor which only depends on the coordinate of the extra dimension, namely

$$
d s_{(5)}^{2}=d w \otimes d w+b^{2}(w) \eta_{\mu \nu} d x^{\mu} \otimes d x^{\nu}
$$

where $b(w)=e^{-k|w|}$ is the warp factor, $\mathrm{k}$ is a constant and $\eta_{\mu \nu}=\operatorname{diag}(-1,1,1,1)$. In their second scenario [].5], where the range of the extra dimension $w$ is $-\infty<w<$ $+\infty$, we live on a four dimensional infinitely thin shell (three brane). Notice that the five dimensional Einstein tensor outside the brane satisfies the Einstein equation with a cosmological constant:

$$
{ }^{(5)} G_{M N}+g_{M N} \Lambda_{5}=0, M, N=0,1,2,3,5
$$

and on $w=$ constant hypersurfaces 4 dimensional Einstein tensor of this metric satisfies

$$
{ }^{(4)} G_{\mu \nu}+g_{\mu \nu} \Lambda_{4}=0, \mu, \nu=0,1,2,3 .
$$

\footnotetext{
*Electronic address: arikm@boun.edu.tr

${ }^{\dagger}$ Electronic address: baykala@boun.edu.tr

¥Electronic address: dciftci@boun.edu.tr

$\S$ Electronic address: odelice@boun.edu.tr
}

where $\Lambda_{5}=-6 k^{2}$ and $\Lambda_{4}=0$. The full Einstein tensor of the 5 dimensional space-time of the metric (10) is given by

$$
{ }^{(5)} G_{M N}=-\eta_{M N} \Lambda_{5}-6 k \delta_{M}^{\mu} \delta_{N}^{\nu} \eta_{\mu \nu} \delta(w) .
$$

Motivated by these considerations, in this work we will calculate five dimensional Einstein equations of the metric (11) for arbitrary $b(w)$ in terms of the four dimensional quantities originating from the four dimensional metric

$$
d s_{(4)}^{2}=g_{\mu \nu} d x^{\mu} \otimes d x^{\nu},
$$

and $b(w)$. As in the Randall-Sundrum scenario we do not wish any matter sources to survive on 5 dimensional space-time except a possible five dimensional cosmological constant. Our most important conclusion will be that a four-dimensional cosmological constant can be induced even when the five-dimensional cosmological constant is zero. We require that only gravity can propagate in extra dimensions. Thus the five dimensional space is an Einstein space where the original Randall-Sundrum metric will be one of the cases of our solutions. Then, as in the Randall-Sundrum scenario we impose reflection $\left(Z_{2}\right)$ symmetry on the extra dimension $w$. This symmetry will make the derivatives of the metric discontinuous with respect to $w$ at the point of symmetry and we know from the thin shell formalism of General Relativity 7] that this discontinuity will give rise to a surface layer (thin shell - brane). The resulting five dimensional Einstein tensor will be of the form (44). Since in our solutions four dimensional part of the metric is same for every $w$, the brane tension (the term proportional to $\delta(w)$ ) is caused only by the jump of $b^{\prime}(w)$ on the brane.

After calculating the five dimensional metric in terms of the four dimensional metric, we first consider the four dimensional cosmological solutions of Einstein equations where the four dimensional space-time is an Einstein space and the four dimensional hypersurface is devoid of matter except a four dimensional cosmological constant. We tabulate all possible solutions we find in Table \

We then consider the four dimensional metric to be given by spherically symmetric static Schwarzschild solution. For this metric we also find all possible solutions $b(w)$ when five dimensional metric is an Einstein space and collect them in Table III Then we will also make some comments on these solutions. 
Our five dimensional metric ansatz can be written in an orthonormal basis as:

$$
\begin{aligned}
d s_{(5)}^{2} & =d w \otimes d w+b(w)^{2} \times\left\{g_{\mu \nu}\left(x^{\rho}\right) d x^{\mu} \otimes d x^{\nu}\right\} \\
& =\eta_{A B} E^{A} \otimes E^{B}
\end{aligned}
$$

where the four dimensional metric is also written in an orthonormal basis:

$$
d s_{(4)}^{2}=g_{i j} d x^{i} \otimes d x^{j}=\eta_{i j} e^{i} \otimes e^{j} .
$$

The orthonormal basis one forms are chosen as

$$
E^{i}=b(w) e^{i}, \quad E^{4}=i b e^{4}=i b d t, \quad E^{5}=d w,
$$

Note that for the sake of computational simplicity, we chose the timelike one form imaginary so that we can take $\eta_{A B}$ as $\delta_{A B}$ and $\eta_{i j}$ as $\delta_{i j}$. The indices run as $A, B, \ldots=$ $1,2,3,4,5$ and $i, j=1,2,3,4$.

Employing Cartan structure equations, we find the nonzero components of the Riemann tensor as follows:

$$
{ }^{(5)} R_{k l}^{i j}=\frac{{ }^{(4)} R_{k l}^{i j}}{b^{2}}-\delta_{k l}^{i j} \frac{b^{\prime 2}}{b^{2}},{ }^{(4)} R^{i 5}{ }_{i 5}=-\frac{b^{\prime \prime}}{b},
$$

where the ' on the functions denote derivatives of the functions with respect to their arguments, and $\delta_{k l}^{i j}$ is generalized Kronecker delta. The Ricci curvature scalar is found as:

$$
{ }^{(5)} R=\frac{{ }^{(4)} R}{b^{2}}-8 \frac{b^{\prime \prime}}{b}-12 \frac{b^{2}}{b^{2}},
$$

Using these one can easily calculate the nonzero components of the Einstein tensor $G_{A B}^{(5)}$ of the metric (6) as:

$$
\begin{aligned}
{ }^{(5)} G_{i j} & =\frac{{ }^{(4)} G_{i j}}{b^{2}}+\delta_{i j}\left\{3 \frac{b^{\prime \prime}}{b}+3 \frac{b^{\prime 2}}{b^{2}}\right\}, \\
{ }^{(5)} G_{55} & =-\frac{R^{(4)}}{2 b^{2}}+\frac{6 b^{\prime 2}}{b^{2}} .
\end{aligned}
$$

We have calculated the nonzero components of the five dimensional Einstein tensor for the metric (6) in terms of $b(w)$ and the four dimensional Einstein tensor of the metric (8). Since we want to first investigate the cosmological solutions we chose four dimensional metric ansatz as follows:

$$
d s_{(4)}^{2}=-d t^{2}+a(t)^{2} d s_{3}^{2}
$$

where

$$
d s_{(3)}^{2}=d \chi^{2}+c(\chi)^{2} d \Omega_{2}^{2}, \quad d \Omega_{2}^{2}=d \theta^{2}+\sin ^{2} \theta d \phi^{2} .
$$

Here we will find admissible values of $b(w), a(t), c(\chi)$ when the Einstein equations satisfy (2) and (3). We can read off the orthonormal basis one forms $e^{i}$ from (14) and (15):

$$
\begin{aligned}
& e^{i}=\left\{e^{4}, a(t) e^{a}\right\}, \quad e^{4}=i d t, \\
& e^{a}=\{d \chi, c(\chi) d \theta, c(\chi) \sin \theta d \phi\}, a, b \ldots=1,2,3 .
\end{aligned}
$$

For (10) the nonzero components of the four dimensional Riemann tensor are found as:

$$
{ }^{(4)} R_{c d}^{a b}=\frac{{ }^{(3)} R_{c d}^{a b}}{a^{2}}+\delta_{c d}^{a b} \frac{\dot{a}^{2}}{a^{2}}, \quad{ }^{(4)} R_{a 4}^{a 4}=\frac{\ddot{a}}{a},
$$

and

$$
{ }^{(3)} R^{12}{ }_{12}={ }^{(3)} R^{13}{ }_{13}=-\frac{\check{c}}{c}, \quad{ }^{(3)} R_{23}^{23}=\frac{1-\check{c}^{2}}{c} .
$$

For the Ricci curvature scalar (11), we have:

$$
{ }^{(4)} R=\frac{{ }^{(3)} R}{a^{2}}+6\left\{\frac{\ddot{a}}{a}+\frac{\dot{a}^{2}}{a^{2}}\right\},{ }^{(3)} R=-4 \frac{\check{c}}{c}+2 \frac{1-\check{c}^{2}}{c^{2}}
$$

The Einstein tensor for this metric (14) is

$$
\begin{aligned}
{ }^{(4)} G_{a b} & =\frac{{ }^{(3)} G_{a b}}{a^{2}}-\delta_{a b}\left\{\frac{2 \ddot{a}}{a}+\frac{\dot{a}^{2}}{a^{2}}\right\}, \\
{ }^{(4)} G_{44} & =-\frac{R^{(3)}}{2 a^{2}}-3 \frac{\dot{a}^{2}}{a^{2}}
\end{aligned}
$$

where

$$
{ }^{(3)} G_{11}=\frac{\check{c}^{2}-1}{c^{2}},{ }^{(3)} G_{22}={ }^{(3)} G_{33}=\frac{\check{\check{c}}}{c} .
$$

Let us combine all these, then ${ }^{(5)} G_{A B}$ becomes:

$$
\begin{aligned}
{ }^{(5)} G_{11} & =\left\{\frac{\check{c}^{2}-1}{a^{2} c^{2}}-\left(2 \frac{\ddot{a}}{a}+\frac{\dot{a}^{2}}{a^{2}}\right)\right\} \frac{1}{b^{2}}+\frac{3 b^{\prime \prime}}{b}+\frac{3 b^{\prime 2}}{b^{2}} \\
{ }^{(5)} G_{22} & =\left\{\frac{\check{c}}{a^{2} c}-\left(2 \frac{\ddot{a}}{a}+\frac{\dot{a}^{2}}{a^{2}}\right)\right\} \frac{1}{b^{2}}+\frac{3 b^{\prime \prime}}{b}+\frac{3 b^{\prime 2}}{b^{2}} \\
& ={ }^{(5)} G_{33} \\
{ }^{(5)} G_{44} & =\left\{\frac{2 \check{c}}{a^{2} c}+\frac{\check{c}^{2}-1}{a^{2} c^{2}}-3 \frac{\dot{a}^{2}}{a^{2}}\right\} \frac{1}{b^{2}}+\frac{3 b^{\prime \prime}}{b}+\frac{3 b^{\prime 2}}{b^{2}} \\
{ }^{(5)} G_{55} & =\left\{\frac{2 \check{c}}{a^{2} c}+\frac{\check{c}^{2}-1}{a^{2} c^{2}}-3\left(\frac{\ddot{a}}{a}+\frac{\dot{a}^{2}}{a^{2}}\right)\right\} \frac{1}{b^{2}}+\frac{6 b^{\prime 2}}{b^{2}} .
\end{aligned}
$$

As we said before, we want to solve these for $a$ and $b$ from ${ }^{(5)} G_{A B}+\delta_{A B} \Lambda_{5}=0$. For ${ }^{(5)} G_{11}={ }^{(5)} G_{22}$ we get the following differential equation:

$$
\frac{\check{c}}{c}=\frac{\check{c}^{2}-1}{c^{2}}
$$

whose set of solutions is

$$
c(\chi)=\left\{\chi, \frac{1}{c_{0}} \sin \left(c_{0} \chi\right), \frac{1}{c_{0}} \sinh \left(c_{0} \chi\right)\right\},
$$

which correspond respectively to the cases $k=0,1,-1$.

For k=0, ${ }^{(5)} G_{i i}={ }^{(5)} G_{44}$ gives the following differential equation:

$$
\frac{\ddot{a}}{a}=\frac{\dot{a}^{2}}{a^{2}}
$$

whose set of solutions is

$$
a(t)=\left\{1 ; e^{a_{0} t}\right\}
$$


For the $a=1$ case, ${ }^{(5)} G_{i i}={ }^{(5)} G_{55}$ gives the equation

$$
\frac{b^{\prime \prime}}{b}=\frac{b^{\prime 2}}{b^{2}}
$$

whose set of solutions is

$$
b(w)=\left\{1 ; e^{b_{0} w}\right\} .
$$

Finally, for the $a=e^{a_{0} t}$ case, we have

$$
\frac{b^{\prime \prime}}{b}=\frac{b^{\prime 2}-a_{0}^{2}}{b^{2}},
$$

whose set of solutions is

$$
b(w)=\left\{a_{0} w ; \frac{a_{0}}{b_{0}} \sinh \left(b_{0} w\right) ; \frac{a_{0}}{b_{0}} \sin \left(b_{0} w\right)\right\} .
$$

In the same way, we can easily find the solutions for $k=\mp 1$. All solutions are shown in Table [] As in the Randall-Sundrum case, to have a brane embedded in five dimensions for these solutions we have to impose $Z_{2}$ symmetry on $b(w)$. Then our four dimensional universe will be an infinitely thin shell at $w=0$ and the total five dimensional Einstein tensor will have of the form:

$$
\begin{aligned}
{ }_{(T)}^{(5)} G_{A B} & ={ }^{(5)} G_{A B}+6 \frac{b^{\prime}}{b} \delta(w) \\
& =-\Lambda_{5} \delta_{A B}-\sigma \delta_{A B} \delta_{\mu}^{A} \delta_{\nu}^{B} \delta(w) .
\end{aligned}
$$

The $k=1$ case corresponds to closed expanding universe with positive cosmological constant, which is in accordance with recent observations [1]. For this case, Table (II) shows that $b(w)$ can take three different values: $\{w ; \sinh w ; \sin w\}$. The first of these is very interesting since in this case the five-dimensional Riemann tensor and the five-dimensional cosmological constant are zero.

To have a $k=1$ solution with ${ }^{(5)} R^{M N}{ }_{P Q}=0$ for this geometry, it is necessary to have nonzero $\Lambda_{4}$. So, flat and empty five-dimensional Minkowski universe in warped geometry (6) can give rise to a four dimensional closed expanding universe with positive cosmological constant. Imposing $Z_{2}$ symmetry, the metric for this case becomes:

$$
\begin{gathered}
d s_{(5)}^{2}=d w^{2}+\left(a_{0}|w|\right)^{2} \\
\times\left\{-d t^{2}+\frac{c_{0}^{2}}{a_{0}^{2}} \cosh ^{2}\left(a_{0} t\right)\left\{d \chi^{2}+\frac{1}{c_{0}^{2}} \sin ^{2}\left(c_{0} \chi\right) d \Omega_{2}^{2}\right\}\right\},
\end{gathered}
$$

with

$$
\begin{aligned}
{ }^{(5)} G_{\mu \nu} & =-6 b^{\prime} / b \delta_{\mu \nu} \delta(w), \\
{ }^{(5)} G_{55} & ={ }^{(5)} G_{5 \mu}=0, \quad{ }^{(4)} G_{\mu \nu}=-\Lambda_{4} \delta_{\mu \nu} .
\end{aligned}
$$

For this case the matter content of the four dimensional universe is only the four-dimensional cosmological constant. In fact, observations show that, the cosmological constant dominates the matter content of the universe. According to the recent review [8], the composition of the content of the universe is as follows:

$$
\begin{aligned}
& \Omega_{B} \approx(0.01-0.2), \quad \Omega_{R} \approx 2 \times 10^{-5}, \\
& \Omega_{D M} \approx 0.3, \quad \Omega_{\Lambda} \approx 0.7,
\end{aligned}
$$

where $\Omega_{B}$ is the density parameter of the visible, nonrelativistic, baryonic matter; $\Omega_{R}$ is the density parameter of the radiation; $\Omega_{D M}$ is the density parameter of the pressureless nonbaryonic dark matter ; and $\Omega_{\Lambda}$ is the density parameter of the cosmological constant. According to the observations which use several independent techniques, the density parameter of the nonrelativistic matter is $\Omega_{N R}=\left(\Omega_{B}+\Omega_{(D M)}\right) \approx(0.2 \sim 0.4)$. This raises the possibility whether with just a four dimensional cosmological constant the five dimensional space-time is flat except on the brane. Other kinds of matter in four dimensions require the five dimensional space-time to fluctuate from flat. Thus the presence of five dimensions differentiates between "dark energy" satisfying equation of state $p=-\rho$ and other forms of matter-energy. Although four dimensional De-Sitter space with positive cosmological constant is consistent with five dimensional flat space, other types of matter-energy in four dimensions require the five dimensional space-time to fluctuate from flatness.

Now we turn to discuss the four dimensional Schwarzschild solution from the five dimensional point of view. Let us choose $d s_{(4)}^{2}$ as Schwarzschild de-Sitter metric which satisfies (3) and is given by:

$$
\begin{aligned}
d s_{(4)}^{2} & =-\left\{1-\frac{2 m}{r}-d_{0} r^{2}\right\} d t^{2} \\
& +\left\{1-\frac{2 m}{r}-d_{0} r^{2}\right\}^{-1} d r^{2}+r^{2} d \Omega_{2}^{2}
\end{aligned}
$$

We find $b(w)$ for $d_{0}<0, d_{0}>0, d_{0}=0$ when the metric (6) satisfies (2) and presented in the Table (III). Note that for $m=0$ and for $d_{0}>0$, The Schwarzschild-de Sitter metric becomes a maximally symmetric metric and this metric can be written in a form where spacelike sections are closed. The metric (34) can be transformed into:

$$
d s_{(4)}^{2}=-d t^{\prime 2}+\cosh ^{2}\left(t^{\prime}\right)\left\{d \chi^{2}+\sin ^{2} \chi d \Omega_{2}^{2}\right\}
$$

with the following transformation

$$
\begin{aligned}
& r=\cosh \left(t^{\prime}\right) \sin (\chi), \\
& t=\ln \left\{\frac{\sinh \left(t^{\prime}\right)+\cosh \left(t^{\prime}\right) \cos (\chi)}{\left\{1-\cosh ^{2}\left(t^{\prime}\right) \sin ^{2}(\chi)\right\}^{1 / 2}}\right\} .
\end{aligned}
$$

For this Schwarzschild-de Sitter case, for $b(w) \sim w$ and $m \neq 0$, five dimensional Riemann tensor is not zero or constant but involves terms proportional to $m / r^{3}$. If $m=$ 0 , the solution reduces to (31). Thus, if we impose $Z_{2}$ symmetry, there will be a brane at $w=0$. Having matter sources on the brane will change the five dimensional metric from flat to curved. Five dimensional Ricci flat but curved metric in warped geometry can give rise to a four dimensional universe with positive cosmological constant and matter. This is a special case of space-time 


\begin{tabular}{|c|c|c|c|c|c|c|c|c|c|c|}
\hline$k$ & $c(\chi)$ & $a(t)$ & $b(w)$ & $R_{N P Q}^{M(5)}$ & $R^{(5)}$ & $\Lambda_{5}$ & $R_{\nu \lambda \kappa}^{\mu(4)}$ & $R^{(4)}$ & $\Lambda_{4}$ & $R^{(3)}$ \\
\hline & \multirow{5}{*}{$\chi$} & \multirow[b]{2}{*}{1} & 1 & 0 & 0 & 0 & 0 & \multirow[b]{2}{*}{0} & \multirow[b]{2}{*}{0} & \multirow{5}{*}{0} \\
\hline & & & $e^{b_{o} w}$ & $-b_{0}^{2}$ & $-20 b_{0}^{2}$ & $-6 b_{0}^{2}$ & 0 & & & \\
\hline 0 & & \multirow{3}{*}{$e^{a_{0} t}$} & $a_{0} w$ & 0 & 0 & 0 & $a_{0}^{2}$ & \multirow{3}{*}{$12 a_{0}^{2}$} & \multirow{3}{*}{$3 a_{0}^{2}$} & \\
\hline & & & $\frac{a_{0}}{b_{0}} \sinh \left(b_{0} w\right)$ & $-b_{0}^{2}$ & $-20 b_{0}^{2}$ & $-6 b_{0}^{2}$ & $a_{0}^{2}$ & & & \\
\hline & & & $\frac{a_{0}}{b_{0}} \sin \left(b_{0} w\right)$ & $b_{0}^{2}$ & $20 b_{0}^{2}$ & $6 b_{0}^{2}$ & $a_{0}^{2}$ & & & \\
\hline \multirow{3}{*}{1} & \multirow{3}{*}{$\frac{1}{c_{0}} \sin \left(c_{0} \chi\right)$} & \multirow{3}{*}{$\frac{c_{0}}{a_{0}} \cosh \left(a_{0} t\right)$} & $a_{0} w$ & 0 & 0 & 0 & $a_{0}^{2}$ & \multirow{3}{*}{$12 a_{0}^{2}$} & \multirow{3}{*}{$3 a_{0}^{2}$} & \multirow{3}{*}{$6 c_{0}^{2}$} \\
\hline & & & $\frac{a_{0}}{b_{0}} \sinh \left(b_{0} w\right)$ & $-b_{0}^{2}$ & $-20 b_{0}^{2}$ & $-6 b_{0}^{2}$ & $a_{0}^{2}$ & & & \\
\hline & & & $\frac{a_{0}}{b_{0}} \sin \left(b_{0} w\right)$ & $b_{0}^{2}$ & $20 b_{0}^{2}$ & $6 b_{0}^{2}$ & $a_{0}^{2}$ & & & \\
\hline \multirow{6}{*}{-1} & \multirow{6}{*}{$\frac{1}{c_{0}} \sinh \left(c_{0} \chi\right)$} & \multirow[b]{2}{*}{$c_{0} t$} & 1 & 0 & 0 & 0 & 0 & \multirow[b]{2}{*}{0} & \multirow[b]{2}{*}{0} & \multirow{6}{*}{$-6 c_{0}^{2}$} \\
\hline & & & $e^{b_{0} w}$ & $-b_{0}^{2}$ & $-20 b_{0}^{2}$ & $-6 b_{0}^{2}$ & 0 & & & \\
\hline & & \multirow{3}{*}{$\frac{c_{0}}{a_{0}} \sinh \left(a_{0} t\right)$} & $a_{0} w$ & 0 & 0 & 0 & $a_{0}^{2}$ & \multirow{3}{*}{$12 a_{0}^{2}$} & \multirow{3}{*}{$3 a_{0}^{2}$} & \\
\hline & & & $\frac{a_{0}}{b_{0}} \sinh \left(b_{0} w\right)$ & $-b_{0}^{2}$ & $-20 b_{0}^{2}$ & $-6 b_{0}^{2}$ & $a_{0}^{2}$ & & & \\
\hline & & & $\frac{a_{0}}{b_{0}} \sin \left(b_{0} w\right)$ & $b_{0}^{2}$ & $20 b_{0}^{2}$ & $6 b_{0}^{2}$ & $a_{0}^{2}$ & & & \\
\hline & & $\frac{c_{0}}{a_{0}} \sin \left(a_{0} t\right)$ & $\frac{a_{0}}{b_{0}} \cosh \left(b_{0} w\right)$ & $-b_{0}^{2}$ & $-20 b_{0}^{2}$ & $-6 b_{0}^{2}$ & $-a_{0}^{2}$ & $-12 a_{0}^{2}$ & $-3 a_{0}^{2}$ & \\
\hline
\end{tabular}

TABLE I: $b(w), a(t), c(\chi)$ and other quantities for 5-d Einstein space when 4-d part is of the form [6

matter (or induced matter) theorem $[9]$ which states that the matter content of the universe is induced from higherdimensional geometry. The relevance of this theorem has been emphasized from the R-S point of view by Wesson and Seahra [10].

In conclusion, we have shown that if in a RandallSundrum like scenario one imposes the condition that $4+1$ dimensional space-time is flat, the only $3+1$ dimensional brane which admits a closed spacelike section cosmology requires a four dimensional cosmological constant. It is clear from Table I that in fact all flat fivedimensional space-time manifolds in warped geometries (6) imply a nonzero and positive cosmological constant for the four-dimensional cosmology. This fact may be important as far as the measured cosmological constant is positive.

\begin{tabular}{|c|c|c|c|c|}
\hline$\Lambda_{4}$ & $b(w)$ & $R^{(5)}$ & $\Lambda_{5}$ & $R^{(4)}$ \\
\hline \multirow{3}{*}{0} & 1 & 0 & 0 & \multirow{3}{*}{0} \\
\cline { 2 - 4 } & $e^{b_{0} w}$ & $-20 b_{0}^{2}$ & $-6 b_{0}^{2}$ & 0 \\
\hline \multirow{3}{*}{$3 d_{0}$} & $w$ & 0 & 0 & \multirow{3}{*}{$12 d_{0}^{2}$} \\
\cline { 2 - 4 } & $\frac{d_{0}}{b_{0}} \sinh \left(b_{0} w\right)$ & $-20 b_{0}^{2}$ & $-6 b_{0}^{2}$ & \\
\cline { 2 - 4 } & $\frac{d_{0}}{b_{0}} \sin \left(b_{0} w\right)$ & $20 b_{0}^{2}$ & $6 b_{0}^{2}$ & \\
\hline$-3 d_{0}$ & $\frac{d_{0}}{b_{0}} \cosh \left(b_{0} w\right)$ & $-20 b_{0}^{2}$ & $-6 b_{0}^{2}$ & $-12 d_{0}^{2}$ \\
\hline
\end{tabular}

TABLE II: $b(w)$ for different signs of $b_{0}$
[1] S. J. Perlmutter, et. al., Nature (London) 391, 51 (1998); A. G. Riess, et. al., Astron. J, 116, 1009 (1998); S. J. Perlmutter, et. al., Astrophys. J, 517, 565 (1999); R. Cen, ibid 509, 16 (1998); L. A. Kofman, N. Y. Gnedin, and N. A. Bahcall, ibid 413, 1 (1993); J. P. Ostriker and P. J. Steinhardt, Nature (London) 377, 600 (1995).

[2] T. Kaluza, Sitzungsber. Preuss. Akad. Wiss., Phys. Math. Kl. K1, 966 (1921); O. Klein, Z. Phys. 37, 895 (1926).

[3] N. Arkani-Hamed, S. Dimopoulos and G. Dvali, Phys Lett. B 429, 263 (1998); I. Antoniadis, N. Arkani-Hamed, S. Dimopoulos and G. Dvali, ibid 436, 257 (1998).

[4] L. Randall and R. Sundrum, Phys. Rev. Lett. 83, 3370
(1999).

[5] L. Randall and R. Sundrum, Phys. Rev. Lett. 83, 4690 (1999).

[6] P. Brax and C. van de Bruck, Class. Quantum Grav. 20, R201 (2003).

[7] W. Israel, Nuovo Cimento B 44, 1 (1966); 48, 463(E) (1967).

[8] T. Padmanabhan, Phys. Rep. 380, 235 (2003).

[9] P. S. Wesson, Space-Time-Matter (World Scientific, Singapore, 1999).

[10] S. S. Seahra and P. S. Wesson, Class. Quantum Grav. 20, 1321 (2003). 